\title{
Interfacial instabilities of a fluid annulus in a rotating Hele-Shaw cell
}

\author{
Lluís Carrillo, Jordi Soriano, and Jordi Ortín ${ }^{\mathrm{a})}$ \\ Departament d'Estructura i Constituents de la Matèria, Facultat de Física, Universitat de Barcelona, \\ Diagonal 647, E-08028 Barcelona, Catalonia (Spain)
}

(Received 16 August 1999; accepted 6 April 2000)

\begin{abstract}
We have studied the interfacial instabilities experienced by a liquid annulus as it moves radially in a circular Hele-Shaw cell rotating with angular velocity $\Omega$. The instability of the leading interface (oil displacing air) is driven by the density difference in the presence of centrifugal forcing, while the instability of the trailing interface (air displacing oil) is driven by the large viscosity contrast. A linear stability analysis shows that the stability of the two interfaces is coupled through the pressure field already at a linear level. We have performed experiments in a dry cell and in a cell coated with a thin fluid layer on each plate, and found that the stability depends substantially on the wetting conditions at the leading interface. Our experimental results of the number of fingers resulting from the instability compare well with the predictions obtained through a numerical integration of the coupled equations derived from a linear stability analysis. Deep in the nonlinear regime we observe the emission of liquid droplets through the formation of thin filaments at the tip of outgrowing fingers. (C) 2000 American Institute of Physics. [S1070-6631(00)01007-2]
\end{abstract}

\section{INTRODUCTION}

Hele-Shaw flows (viscous flows in the gap between two closely spaced parallel plates) have been the subject of numerous studies in recent years. From a practical point of view, the interest in these problems arises from the fact that Hele-Shaw flows are governed by the same equations as flows in porous media. In particular, Hele-Shaw flows exhibit a viscous fingering instability, ${ }^{1}$ which is believed to determine the efficiency of oil recovery processes by water displacement in porous oil reservoirs, and is also present in underground storage of gas. ${ }^{2}$ Another related problem of practical interest is injection molding, in which a fluid displaces air in a mold which often has the same parallel plate geometry as Hele-Shaw cells. The displacement in this case is stable, and the question is to define the conditions for complete elimination of air in the mold. The study of HeleShaw flows driven by body forces (gravity or centrifugal) may also be of interest in the technology of coating. ${ }^{3}$

On the other hand, the viscous fingering instability experienced by Hele-Shaw flows is regarded from a fundamental point of view as a relatively simple but very rich scenario to study generic features of interfacial pattern formation in dynamic nonlinear systems. ${ }^{4,5}$ The SaffmanTaylor problem, as commonly known in this context, is a prototypical example of a moving-boundary problem, relevant to a broad class of morphological instabilities found not only in porous media flows, but also within the context of crystal growth, electrochemical deposition, dielectric breakdown, and flame propagation, for example. ${ }^{5}$

A number of modifications of the basic Saffman-Taylor problem have been considered in recent years. ${ }^{6}$ An interesting possibility is to impose a uniform rotation about an axis

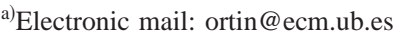

perpendicular to the plane of the flow, in the circular geometry. The morphological instability induced by centrifugal forcing is driven by the density difference and not by the viscosity difference of the two fluids. The linear stability analysis of this problem in the limits of high density and viscosity contrast was worked out by Schwartz, ${ }^{8}$ and extended to arbitrary density and viscosity contrast by our group. ${ }^{9}$ In this latter work we verified experimentally the maximum growth rate selection of initial patterns within experimental uncertainty, and also studied the nonlinear regime in the case of vanishing injection rate.

A particularly interesting arrangement in the rotating cell is that of a liquid annulus, centered in the cell, which defines three different regions separated by two interfaces: A trailing or inner interface $(i)$, and a leading or outer interface $(o)$. Depending on densities and viscosities of the fluids in the different regions, and on the experimental parameters selected (gap thickness, initial volume, and rotational frequency), qualitatively different scenarios of interfacial instabilities can be considered. In this paper we study an annular arrangement in the limits of high density and viscosity contrast, where air occupies the innermost and outermost layers, and oil occupies the intermediate layer. Emphasis is laid on the general situation in which either one of the two or both interfaces are unstable, with particular interest on the behavior resulting from their coupled motion.

Related to this study, three-fluid annular Hele-Shaw flows with no centrifugal forcing were recently considered by Cardoso and Woods. ${ }^{10}$ In their work the radial spreading of the fluids is forced by the steady injection of fluid in the innermost layer. The emphasis is on the behavior when one of the interfaces is highly stable and the other is unstable. In this configuration, the authors report that surface tension at small radii and the continuous thinning of the intermediate layer at large radii stabilize the system. 


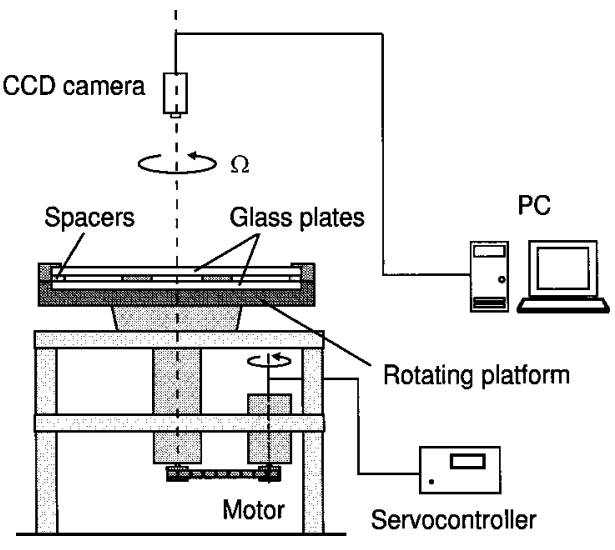

FIG. 1. Sketch of the experimental apparatus.

The work presented in this paper completes our study of the problem of a fluid annulus in a rotating Hele-Shaw cell, which was initiated in Ref. 11 with the study of stable displacements. Centrifugal forcing allows a wide range of driving force amplitudes. This led us to study annular flows in a wide range of capillary numbers, covering about three orders of magnitude. The study revealed the relevance of wetting conditions to the behavior of the flow; the interfaces of the annulus moved with monotonously rising velocities in a cell prewet with a thin fluid layer on each glass plate, and with much lower constant velocities in a dry cell. ${ }^{11}$ The consequences of this different behavior on the instability thresholds of the two interfaces of the annulus constitute the central part of the study presented here.

In a rotating cell, the bulk pressure due to centrifugal forcing increases linearly in the radial direction. This enhances secondary bifurcations and the occurrence of topological singularities in the flow, such as the formation of fluid filaments and the breakup of the interface into liquid droplets. We report on qualitative observations of such phenomena in our experiments.

In summary, we present a linear stability analysis and an experimental study of the fingering instabilities that occur during the radial displacement of a spinning fluid annulus in a Hele-Shaw cell, for a wide range of experimental parameters (fluid volume, rotational frequency, gap thickness), different wetting conditions (prewet and dry), and different properties of the liquid (viscosity, density, and surface tension).

\section{EXPERIMENT}

\section{A. Experimental apparatus}

Our Hele-Shaw cell is made of two $6 \mathrm{~mm}$ thick circular glass plates of $40 \mathrm{~cm}$ diam, separated by six calibrated spacers of thicknesses $b$ in the range $0.25-2.00 \mathrm{~mm}$, and firmly clamped together. We estimate the fluctuations in gap spacing at about $0.05 \mathrm{~mm}$.

The cell is mounted on top of a rotating cylindrical platform, as shown in Fig. 1. To accurately balance the cell even at high angular velocities and to damp vibrations, the axis of the platform is conical and sits on a large conical ball bearing attached to the supporting structure. This structure rests on a

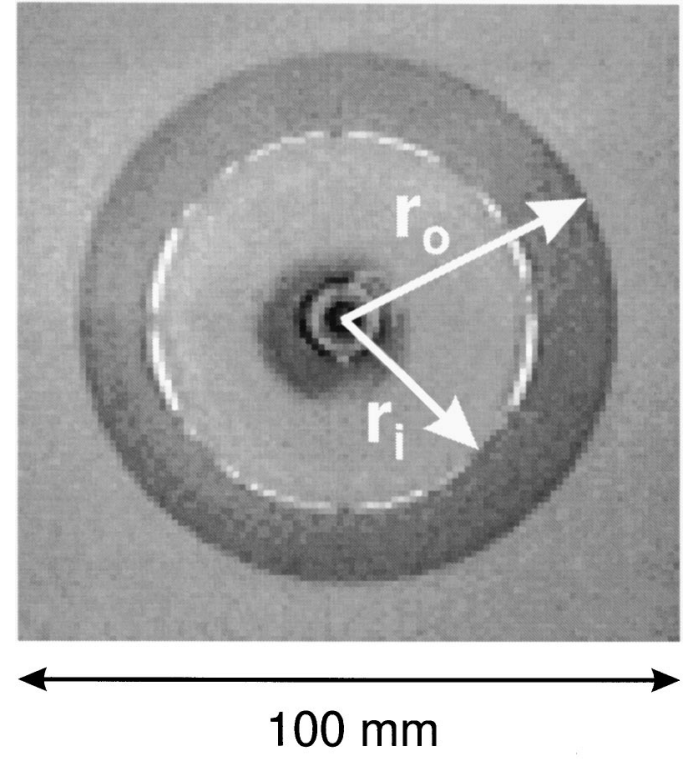

FIG. 2. Digital picture of a liquid annulus (inner radius $r_{i}$, outer radius $r_{o}$ ) extracted from a sequence of video frames. The dark central spot is a reflection of the camera lens on the glass plate, overlapped with the central orifice of the plate. The picture is representative of the resolution achieved with our image acquisition setup.

heavy granite table with three adjustable feet, in order to accurately level the cell, which is critical during rotation. Before each run, the center of the cell is carefully aligned with the rotation axis, to a tolerance less than $0.02 \mathrm{~mm}$ in the radial direction.

The axis of the platform is coupled via a belt to a pulley mounted on the shaft of a variable speed motor, equipped with a reductor and a tachometer. The signal from the tachometer is used by an external servocontroller to maintain a highly constant rotation speed $\Omega$, independently of load variations, in the range $0-300 \mathrm{rev} / \mathrm{min}(0-5 \mathrm{~Hz})$ with fluctuations around $\pm 0.1 \mathrm{rev} / \mathrm{min}$. We have found empirically ${ }^{11}$ that the rotation speed of the platform, when it accelerates from rest to a preset value $\Omega_{0}$, is well described by $\Omega$ $=\Omega_{0}[1-\exp (-\omega t)]$, where $\omega=1.2\left(300 / \Omega_{0}\right)^{2.5}$ and both $\Omega$ and $\Omega_{0}$ are given in rev/min. Thus, the platform accelerates to $50,100,200$, and $300 \mathrm{rev} / \mathrm{min}$ in less than $0.04,0.4,2$, and $6 \mathrm{~s}$, respectively. Except for a few exceptions, these transient times are negligible in most experiments. The maximum deflection of the upper glass plate due to the rotation of the cell is $0.03 \mathrm{~mm}$ at $300 \mathrm{rev} / \mathrm{min}$.

Four white fluorescent tubes, arranged forming a square around the cell at the level of the gap, constitute the light source. The change in refraction index as the light passes from air to liquid or vice versa renders the interfaces visible. An example is shown in Fig. 2. Images are recorded with a JVC TK-S340 CCD camera, equipped with a $8 \mathrm{~mm}$ Cosmicar Pentax objective, placed above the apparatus and focused on the glass plates. Sharp images of patterns rotating at high speed are obtained using the electronic shutter of the camera, which allows pictures to be taken in 1/10000 s. The camera is connected to a Vitec VideoMaker frame grabber installed in a personal computer, which digitizes the images sequentially and stores them in memory. The spatial resolution of 
TABLE I. Properties of the fluids used in the experiments.

\begin{tabular}{lcccc}
\hline \hline & & $\begin{array}{c}\text { Silicone } \\
\text { oil } 50\end{array}$ & $\begin{array}{c}\text { Silicone } \\
\text { oil 500 }\end{array}$ & $\begin{array}{c}\text { Vaseline } \\
\text { oil } 150\end{array}$ \\
\hline Kinematic viscosity $\nu\left(\right.$ at $\left.25^{\circ} \mathrm{C}\right)$ & $\mathrm{mm}^{2} / \mathrm{s}$ & 50 & 500 & 150 \\
Density $\rho$ & $\mathrm{kg} / \mathrm{m}^{3}$ & 998 & 998 & 870 \\
Oil-air interfacial tension $\sigma$ & $\mathrm{mN} / \mathrm{m}$ & 20.7 & 20.7 & 29.3 \\
\hline \hline
\end{tabular}

the images is fixed to $288 \times 288$ pixels, and the capture rate is varied between 0.25 and 6 images/s, with 256 gray levels per pixel. The relative orientation between successive images is determined by a number of markers on the top glass plate.

\section{B. Experimental procedure}

We have used two silicone oils (Rhodorsil) of the same density and surface tension, and very different viscosity, and a vaseline oil (Panreac) of intermediate viscosity, lower density and higher surface tension. The properties of the liquids are summarized in Table I. The viscosities were measured using a Cannon-Fenske capillary viscometer. The interfacial tension of the vaseline oil was measured using the pendant drop method, after having checked the procedure on the two silicone oils, for which data were available. The three liquids are transparent. They have been chosen for their Newtonian behavior and for perfectly wetting the glass.

We have taken good care in properly cleaning the cell after each experiment. First, oil traces have been wiped off with paper. Next, the two plates have been thoroughly cleaned with soap and water, rinsed with distilled water and acetone, and finally dried with pressurized air. Since the liquids used in the present experiments are rather insensitive to substrate contamination, we have found this protocol sufficient to obtain reproducible surface conditions.

In our work on the radial displacement of the annulus ${ }^{11}$ we reported on the significant influence that wetting conditions at the leading interface have on the properties of the flow. To further study this problem, this time in connection with the stability of the annulus interfaces, the same two experimental procedures have been followed:

Preparation of the annulus in a dry cell.

In the first procedure the displacement takes place in $d r y$ conditions. The rotation speed of the motor is first preselected. The liquid is injected into the motionless cell (which has been cleaned as described above) using a syringe pump through a central orifice of radius $R_{A}=4 \mathrm{~mm}$ machined in the upper plate. The liquid droplet formed is allowed to grow until it reaches a prescribed radius $L_{0}$. At this moment the injection tube is removed and the motor is switched on. Since air enters the cell through the open central orifice, the liquid droplet quickly becomes a liquid annulus which spreads radially, and eventually destabilizes, giving rise to a fingering pattern. The process is digitally recorded until the pattern reaches the edge of the cell.

(ii) Preparation of the annulus in a prewet cell.

In the second procedure the aim is to prepare the cell with a thin liquid layer deposited on each glass plate.
To this end, oil is slowly injected into the motionless cell up to a radius of about $100 \mathrm{~mm}$, the injection tube is disconnected, and the platform is set to rotate at low speed (between 40 and $60 \mathrm{rev} / \mathrm{min}$ ). The liquid droplet becomes an annulus, which slowly spreads radially and finally disappears when the trailing interface reaches the edge of the cell. The motor is then switched off. This method produces an oil layer of relative thickness 0.1 on each glass plate for a rotation speed of $50 \mathrm{rev} / \mathrm{min}$. In the cell prewetted in this way, a new liquid droplet is formed immediately up to a radius $L_{0}$ by additional oil injection. The rest of the procedure follows the same steps of the dry cell.

\section{Experimental results}

Our experiments have covered a large region of the available parameter space. The experimental parameters have centered around the following predetermined values: $b=0.25,0.50,0.81,1.0$, and $1.94 \mathrm{~mm} ; \Omega=30,40,50,60$, $80,90,120,150,180,210,240,270$, and $300 \mathrm{rev} / \mathrm{min} ; L_{0}$ $=10,16,20,32,49$, and $95( \pm 1) / \mathrm{mm}$.

The experiments show that the radial displacement of the annulus in prewet conditions is stable up to very large radii. The interface velocities rise in time. If the amount of liquid (measured by $L_{0}$ ) is sufficiently large to prevent the two interfaces from meeting each other during the thinning process experienced by the annular layer, the interfaces remain stable until the liquid leaves the cell at the outer edge (Fig. $3)$. For smaller amounts of liquid ( $L_{0}$ smaller) the interfaces remain stable until they get so close to each other that the annulus becomes extremely thin, and visually seems to vanish on the glass plates when the meniscii of the two interfaces pinch.

The displacement of the annulus in dry conditions is generally unstable. The circular interfaces move at nearly constant velocity, sensibly lower than in equivalent experiments in prewet conditions (Fig. 4), until fingering instabilities develop. The number of ripples generated depend on the experimental parameters. Most combinations of parameters make the leading interface destabilize first. It is also common to have both interfaces destabilizing simultaneously when the motion of the two interfaces is strongly coupled, particularly for small $L_{0}$. It is only for very large $L_{0}$ that the instabilities develop first at the trailing interface.

The examples shown in Fig. 5 correspond to experiments with silicone oil 50 in dry conditions. In Fig. 5(a), although both interfaces become unstable, the leading interface destabilizes first. In Fig. 5(b), on the other hand, the two interfaces become unstable at the same time with the same dominant wave number. This is a case in which the motion of the interfaces is strongly coupled because $r_{o} \simeq r_{i}$. Although the thickness of the annulus, about $3 \mathrm{~mm}$, is still larger than the gap thickness, $b=0.81 \mathrm{~mm}$, threedimensional effects begin to play a role and this experiment approaches the limit of validity of the Hele-Shaw regime. In Fig. 5(c), which corresponds to very large $L_{0}$, the trailing interface destabilizes first, and it is only when this instability has developed far beyond the linear regime that the leading 

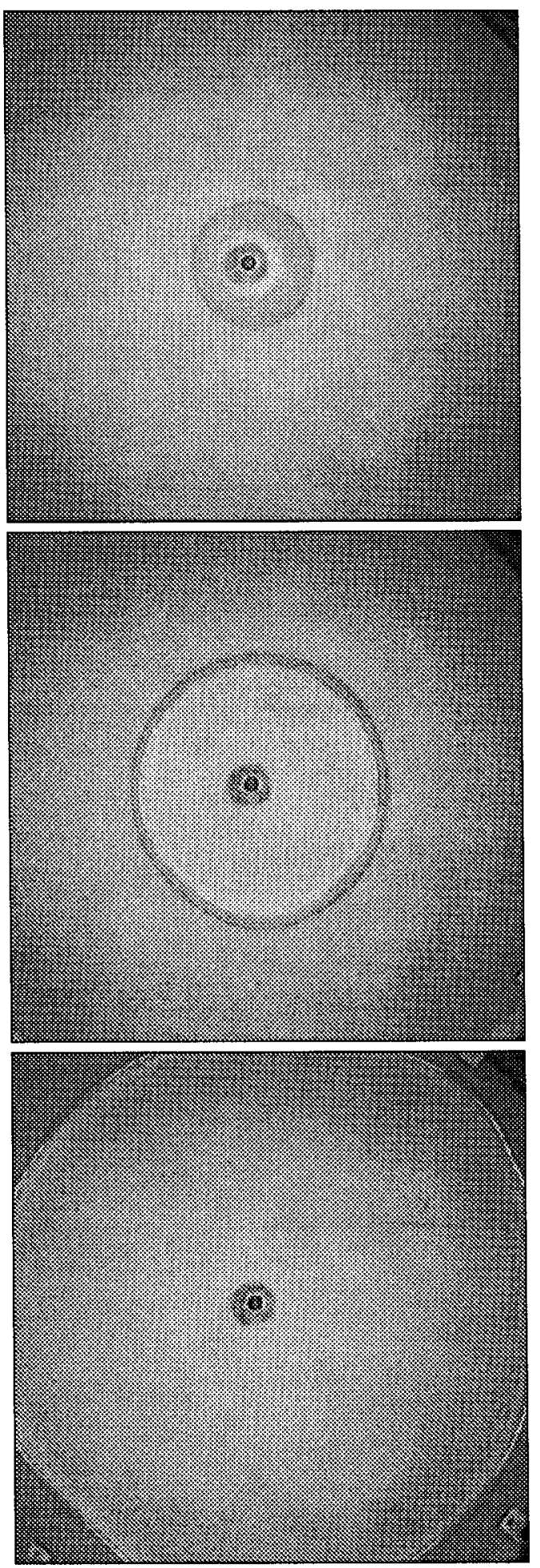

FIG. 3. Displacement of an annular layer of silicone oil 50 in a prewet cell. The interfaces are stable during the displacement until they reach the edge of the cell. The experimental parameters are $L_{0}=30 \mathrm{~mm}, \Omega=60 \mathrm{rev} / \mathrm{min}$, and $b=0.81 \mathrm{~mm}$. The time interval between consecutive pictures (from top to bottom) is $25 \mathrm{~s}$.

interface shows signs of destabilization, with a dominant wave number independent of that developed at the trailing interface. In this case the motion of the interfaces is practically uncoupled, since $r_{o} \gg r_{i}$.

The examples shown in Fig. 6 correspond to experi-
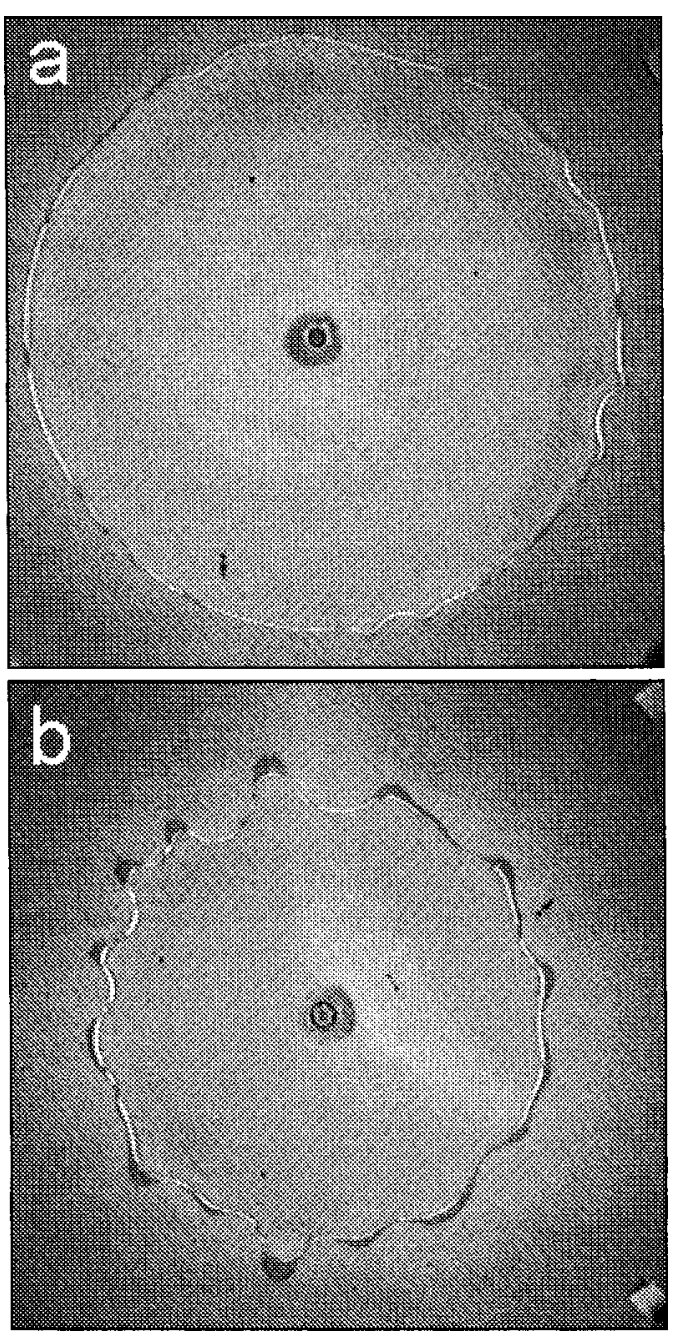

FIG. 4. Displacement of an annulus of silicone oil 50 in a prewet cell (a), and a dry cell (b), showing the influence of wetting conditions on the stability of the interfaces. In the two experiments the parameters are $L_{0}$ $=30 \mathrm{~mm}, \Omega=120 \mathrm{rev} / \mathrm{min}$, and $b=0.81 \mathrm{~mm}$. The pictures have been taken at $t=13.2 \mathrm{~s}$ in both cases.

ments with vaseline oil 150 in dry conditions. Here the only parameter modified is $L_{0}$, which increases from top to bottom. For small $L_{0}$ (on top) the two interfaces become unstable almost simultaneously and with the same dominant wave number. In the experiment shown here the perturbations at the two interfaces are in phase, but in a few cases we observed perturbations in phase opposition. For an intermediate value of $L_{0}$ (in the middle) the leading interface is observed to destabilize first, while the trailing interface remains nearly perfectly circular for some time, as shown in the picture. In most cases this interface finally also destabilizes, with a similar dominant wavenumber. Finally, for large $L_{0}$ (at the bottom) we observe a situation very similar to that shown in Fig. 5(c) in which the instability develops first at the trailing interface. The leading interface, in spite of having a very large radius, remains stable through most of the displacement.

The behavior of the dominant wavenumber $n$ emerging from the linear regime with $L_{0}$ is the following: in most cases the instability develops first at the leading interface, 

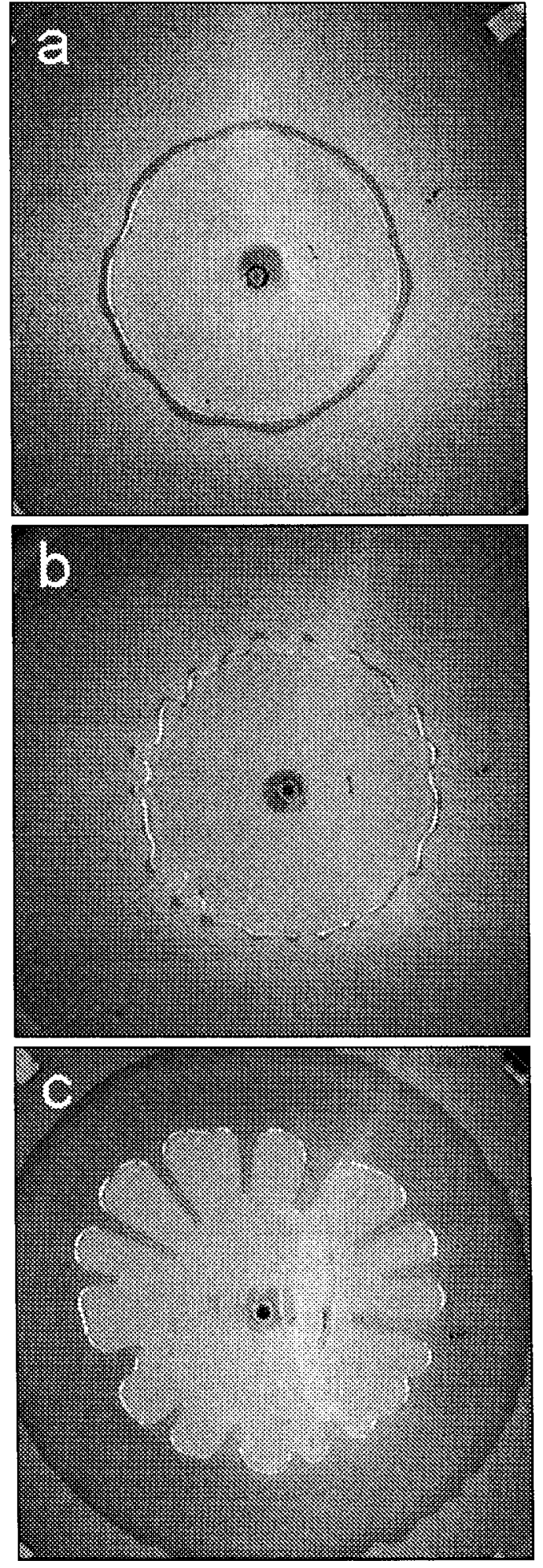

FIG. 5. Interfacial instabilities of an annulus of silicone oil 50, in a dry cell of gap spacing $b=0.81 \mathrm{~mm}$, for different initial radii and rotation speeds: (a) $L_{0}=30 \mathrm{~mm}, \Omega=90 \mathrm{rev} / \mathrm{min}$, (b) $L_{0}=30 \mathrm{~mm}, \Omega=210 \mathrm{rev} / \mathrm{min}$, (c) $L_{0}$ $=117 \mathrm{~mm}, \Omega=120 \mathrm{rev} / \mathrm{min}$.

and $n$ increases with $L_{0}$. Above a given $L_{0}$, however, the instability develops first at the trailing interface, the value of $n$ drops, and then (for the limited parameter range that remains accessible) increases again with $L_{0}$.

The time evolution of the interfaces is obtained from the
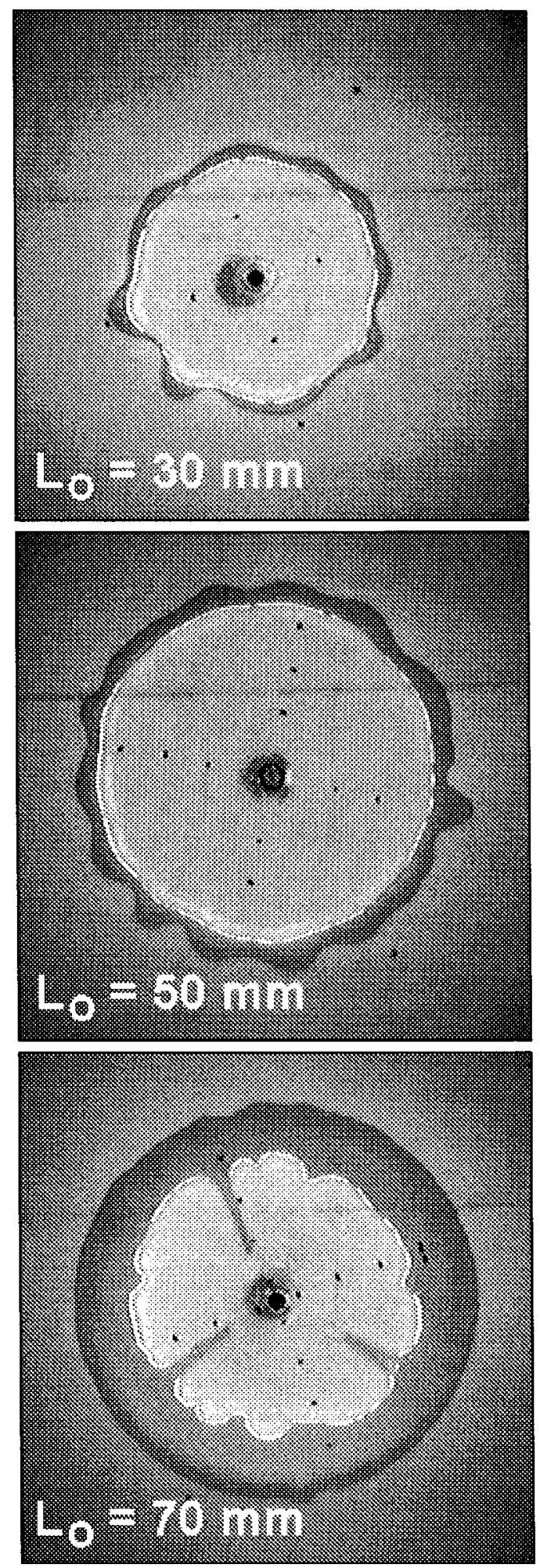

FIG. 6. Interfacial instabilities of an annulus of vaseline oil 150, in a dry cell of gap thickness $b=0.81 \mathrm{~mm}$ rotating at $\Omega=180 \mathrm{rev} / \mathrm{min}$. The initial radius of the drop in each run, $L_{0}$, increases from top to bottom.

sequence of digital video images, captured at equal time intervals. Using edge detection techniques, we have determined the line $r(s)$ for each interface. This line gives the radial extent of the interface as a function of the interface arclength. Use of the arclength instead of the azimuthal angle $\theta$ makes no substantial difference in the linear regime. In the 


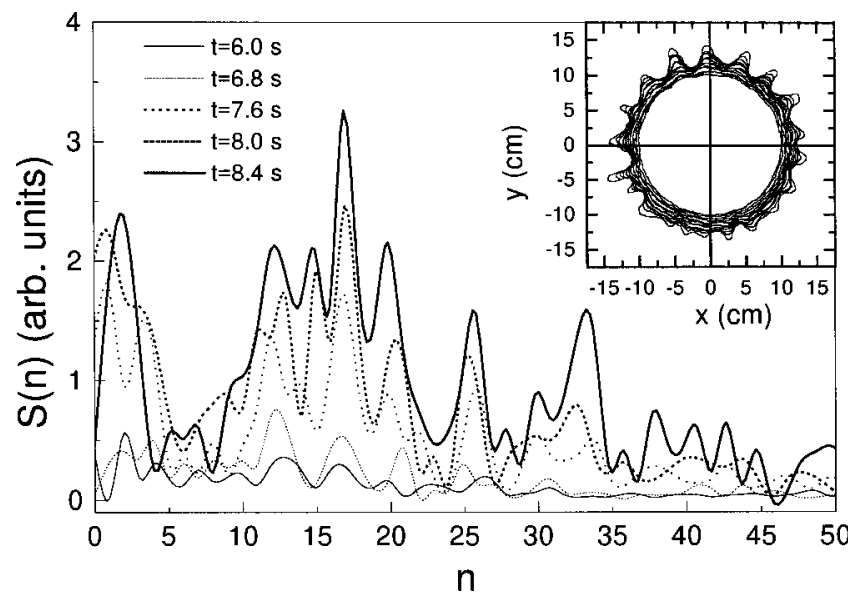

FIG. 7. Evolution of the power spectrum of a leading interface at the onset of instability, demonstrating the development of modes of larger $n$ as time progresses. The inset shows the sequence of leading interfaces digitized at times $6.0,6.2,6.4,6.6,6.8,7.0,7.4,7.6,7.8,8.0$, and $8.4 \mathrm{~s}$. The fluid is silicone oil 50, in a dry cell, and the experimental parameters are $b$ $=0.81 \mathrm{~mm}, \Omega=150 \mathrm{rev} / \mathrm{min}$, and $L_{0}=31 \mathrm{~mm}$.

nonlinear regime, however, it eliminates the problems associated with the possibility that $r(\theta)$ becomes multivalued. The onset and development of the instability have been investigated by Fourier mode analysis. To this end, for each digitized interface $r(s)$ we have computed the function

$$
\rho(s)=r(s)-R,
$$

where $R$ is the radius of a circle obtained from a leastsquares fit of $r(s)$. The function $\rho(s)$ represents the deformation of the interface line with respect to this circle. The discrete Fourier transform of $\rho(s)$ is given by

$$
\hat{\rho}(n)=\sum_{j=0}^{N-1} \rho(2 \pi j / N) e^{i n 2 \pi j / N},
$$

where $n$ is the azimuthal wave number and $N$ is the number of data points in the digitized curve $\rho(s)$.

The power spectrum of the leading interface of an annulus of silicone oil 50, computed at five successive instants, is shown in Fig. 7. At the earliest time the amplitude of the maximum at $n=2$ is of the order of the noise in the spectrum, and no dominant mode can be identified. As time advances, the maximum in the spectrum shifts to $n=12$ and then to $n=17$. Competing with this mode $n=17$ there is a mode at $n=20$, which grows at a similar or higher rate, and which can be identified with the number of fingers developing at later times, already in the large amplitude regime. Some harmonics (at $n=34$ in particular) and other modes of smaller amplitude also appear.

The evolution towards modes of larger wave number, revealed by this numerical analysis, cannot be easily derived by a visual inspection of the digitized interfaces. The time sequence shown in the inset contains a total of eleven interfaces, of which the first (still practically unperturbed), the fifth, the eighth, the tenth, and the last (slightly beyond the linear regime) have been used in the Fourier analysis. Inspection of the latter interface confirms that the number of fingers is $\sim 20$. It is important to mention that this number remains

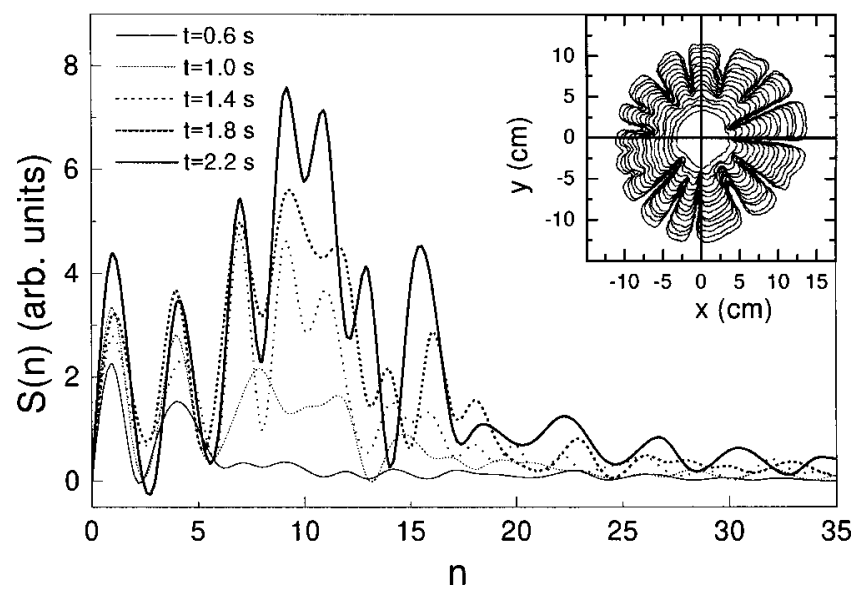

FIG. 8. Evolution of the power spectrum of an unstable trailing interface. The inset shows fifteen interfaces digitized every $0.2 \mathrm{~s}$, starting at $t$ $=0.6 \mathrm{~s}$. The fluid is silicone oil 50, in a dry cell, and the experimental parameters are $b=0.81 \mathrm{~mm}, \Omega=120 \mathrm{rev} / \mathrm{min}$, and $L_{0}=117 \mathrm{~mm}$.

unchanged for the rest of the experiment, because deep in the large amplitude regime there is no sign of any mechanism (tip splitting or finger competition) capable of modifying the number of fingers growing out of the leading interface. The reason must be found in the increasing lateral separation between radially growing fingers in the circular geometry, and in the fact that pressure grows with the square of the radial distance in the presence of centrifugal forcing.

The same analysis has been performed on a trailing interface, as shown in Fig. 8. In early stages the maximum of the power spectrum is at the mode $n=1$, which represents a circular interface slightly off axis. Above $1.0 \mathrm{~s}$ the modes $n=7$ and $n=9$ take off with similar amplitude. Finally, at the latest time shown $(2.2 \mathrm{~s})$ the mode with $n=9$ has reached the maximum amplitude. The lower amplitude modes $n$ $=11$ and $n=16$, however, are seen to grow at similar rates, and presumably will take over from lower $n$ modes at later times.

For this case we have chosen a time sequence of digitized interfaces (inset) extending deep in the large amplitude regime. Out of the fifteen interfaces shown, the power spectrum covers the time interval from the first to the ninth interface, which is already beyond the linear regime. As opposed to the behavior of the leading interface, deeper in the nonlinear regime the time evolution of the fingering pattern of the trailing interface is characterized by a growing number of fingers, due to finger splitting at the tip of wide fingers. This behavior is characteristic of viscosity driven instabilities in the radial geometry. Notice that the last interface shown displays about fifteen well-developed fingers, but this number changes to about eighteen fingers due to tip splitting.

\section{ANALYSIS AND DISCUSSION}

In this Section, we review the Hele-Shaw equations for the annular geometry in the presence of centrifugal forcing, and perform a linear stability analysis of the interfaces. This provides the theoretical background required to guide the analysis of our experimental results. 


\section{A. Hele-Shaw equations}

We consider an oil annulus of inner radius $r_{i}$ and outer radius $r_{o}$, in a circular Hele-Shaw cell of plate spacing $b$ rotating with angular velocity $\Omega$. In the high-friction limit (Hele-Shaw approximation) the velocity field in the bulk of the liquid, averaged in the direction perpendicular to the plates, obeys Darcy's law

$$
\mathbf{v}=\nabla \phi,
$$

where

$$
\phi=-M\left(p-\frac{1}{2} \rho \Omega^{2} r^{2}\right),
$$

and the mobility $M=b^{2} / 12 \mu$. Here $\mu$ is the dynamic viscosity and $\rho$ is the density of the oil. Since the liquid is incompressible, $\nabla \cdot \mathbf{v}=0$, and Darcy's law leads to a Laplace equation for the velocity potential

$$
\nabla^{2} \phi=0 .
$$

The nonlinearities enter through the boundary conditions at the interfaces.

The first set of boundary conditions specifies the pressure jump at the two interfaces

$$
\begin{aligned}
& \frac{\phi\left(r_{i}\right)}{M}-\frac{1}{2} \rho \Omega^{2} r_{i}^{2}=\sigma\left(\kappa_{\perp_{i}}+\kappa_{\|_{i}}\right), \\
& \frac{\phi\left(r_{o}\right)}{M}-\frac{1}{2} \rho \Omega^{2} r_{o}^{2}=\sigma\left(\kappa_{\perp_{o}}-\kappa_{\|_{o}}\right),
\end{aligned}
$$

where $i$ and $o$ refer to the inner and outer interface, respectively, and $\kappa_{\perp}, \kappa_{\|}$are the curvatures of the interface in the directions perpendicular and parallel to the plates, respectively. In writing these boundary conditions we have neglected the contribution of air to the pressure difference.

The second set of boundary conditions refers to the continuity of the normal velocity across the interfaces

$$
\begin{aligned}
& \left.\partial_{r} \phi_{1}\right|_{i}=\left.\partial_{r} \phi_{2}\right|_{i} \cdot \frac{1}{1-\alpha_{i}}, \\
& \left.\partial_{r} \phi_{1}\right|_{o}=\left.\partial_{r} \phi_{2}\right|_{o} \cdot \frac{1}{1-\alpha_{o}},
\end{aligned}
$$

where $\alpha_{i}, \alpha_{o}$, are the relative thicknesses of the liquid films on the glass plates, behind the leading interface and ahead of the trailing interface respectively (see Ref. 11).

When the two interfaces are circular, the solution of Eq. (5) for the velocity potential has the form

$$
\phi^{0}(r)=A \ln r+B,
$$

where conditions (6) and (7) determine the constant $A$ as

$$
A=M \frac{(1 / 2) \rho \Omega^{2} L^{2}-\sigma\left[(2 / b)\left(\cos \theta_{D i}-\cos \theta_{D o}\right)+1 / r_{i}+1 / r_{o}\right]}{\ln \left(r_{o} / r_{i}\right)} .
$$

Here $L^{2} \equiv r_{o}^{2}-r_{i}^{2}$, and initially $L^{2}=L_{0}^{2}$. We have taken $\kappa_{\perp i, o}=(2 / b) \cos \theta_{D i, o}$ and $\kappa_{\| i, o}=1 / r_{i, o}$, where $\theta_{D i, o}$ represent the dynamic contact angles at the two interfaces. The constant $B$ is determined by prescribing the magnitude of the pressure at some point in the flow. The index 0 in $\phi^{0}$ is a reminder that this solution is valid for an annulus with unperturbed circular interfaces.

\section{B. Influence of wetting}

In our first paper on the problem of the annulus ${ }^{11}$ we focused on stable (radial) displacements. We found that these displacements are very sensitive to the prewet or dry conditions of the cell, and identified the two main phenomena responsible for this:

(i) The dynamic contact angles at the interfaces experience variations in the range 0 to $\pi$, depending on their normal velocity. This makes the perpendicular curvatures independently variable in the range $2 / b$ to $-2 / b$. If the curvatures remain the same at the two interfaces, as is approximately the case in a prewet cell, the corresponding jumps of capillary pressure at the interfaces cancel out. If not, as in a dry cell, the jumps of capillary pressure may be very different and have a relevant influence on the radial velocity.

(ii) In both prewet and dry cells the trailing interface leaves a liquid layer on the two glass plates. The thickness of the layer depends on the normal velocity of the interface. The thickness was measured by a stable displacement of the annulus in Ref. 11 (Fig. 8). In a dry cell, the formation of the layer results in a progressive loss of liquid in the annulus. In a prewet cell, this loss is largely compensated at the leading interface by the liquid regained from the film coating the glass plates. As a result, $L^{2}$ (the amount of liquid in the annulus), decreases with time in a dry cell and stays almost constant in a prewet cell.

It is important to note that the two phenomena depend on the instantaneous radial velocity of the interfaces.

The experiments confirmed that the approximation of perfect wetting, $\kappa_{\perp i} \simeq \kappa_{\perp o}$ and $L^{2} \simeq L_{0}^{2}$, provides an accurate description of stable displacements in prewet conditions. In this case, the velocities of the two interfaces scaled as predicted by the above equations. ${ }^{11}$

On the other hand, for stable displacements in a dry cell a nontrivial result arises; the velocity of the two interfaces is nearly constant throughout the displacement. The velocity seems to be dynamically selected by the interplay of the two phenomena considered above, both dependent on interface velocity. Defining the capillary number for constant velocity displacements as $C a=\mu v / \sigma$, and the ratio of centrifugal to capillary forces as $S=\rho \Omega^{2} L_{0}^{3} / \sigma$, our experiments showed that in dry conditions $C a$ scales with $\left(b / L_{0}\right)^{2} S^{5 / 4}$ for about three orders of magnitude of the two quantities. ${ }^{11}$

\section{Linear stability analysis of the fingering instability}

To carry out a linear stability analysis of the interfaces, we write the perturbative equations in their simplest form, without including corrections due to wetting. We assume that it is enough to account for these corrections on the unperturbed velocity field. 


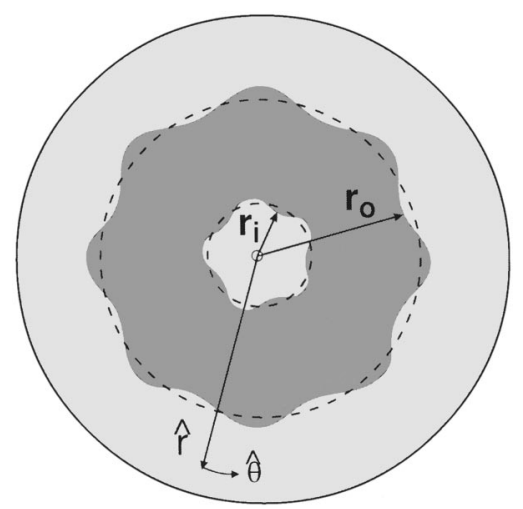

FIG. 9. Perturbation of the two circular interfaces of a liquid annulus.

Let us modify the two circular interfaces instantaneously (Fig. 9) by adding an infinitesimal perturbation of the form

$$
\begin{aligned}
& \delta r_{i}=\zeta_{i} e^{i n \theta}, \\
& \delta r_{o}=\zeta_{o} e^{i m \theta},
\end{aligned}
$$

to each interface, respectively. The ansatz for the velocity potential of the perturbed annulus takes the form

$$
\phi(r, \theta)=\phi^{0}(r)+\phi_{i}^{1}(r) e^{i n \theta}+\phi_{o}^{1}(r) e^{i m \theta},
$$

where

$$
\phi_{i}^{1}(r)=\frac{C_{n}}{r^{n}}+D_{n} r^{n}, \quad \phi_{o}^{1}(r)=\frac{C_{m}}{r^{m}}+D_{m} r^{m} .
$$

Adding the condition that the perturbation at one interface does not affect the other:

$$
\phi_{i}^{1}\left(r_{o}\right)=0, \quad \phi_{o}^{1}\left(r_{i}\right)=0,
$$

leads to:

$$
C_{n}=-D_{n} r_{o}^{2 n}, \quad C_{m}=-D_{m} r_{i}^{2 m} .
$$

The constants $D_{n}, D_{m}$ are determined by the boundary conditions (6) and (7). Taking into account the fact that $\phi^{0}(r)$ itself satisfies these boundary conditions for the circular interfaces $\left(r=r_{i}, \quad \kappa_{\| i}=1 / r_{i}\right.$, and $r=r_{0}, \kappa_{\| o}=1 / r_{o}$, respectively) and linearizing the curvatures in the parallel direction, we obtain

$$
\begin{gathered}
\left.\frac{1}{M} \partial_{r} \phi^{0}\right|_{r_{i}} \delta r_{i}-\rho \Omega^{2} r_{i} \delta r_{i}+\frac{1}{M} \phi_{i}^{1}\left(r_{i}\right) e^{i n \theta} \\
=\frac{\sigma}{r_{i}^{2}}\left(n^{2}-1\right) \delta r_{i}, \\
\left.\frac{1}{M} \partial_{r} \phi^{0}\right|_{r_{o}} \delta r_{o}-\rho \Omega^{2} r_{o} \delta r_{o}+\frac{1}{M} \phi_{o}^{1}\left(r_{o}\right) e^{i m \theta} \\
=-\frac{\sigma}{r_{o}^{2}}\left(m^{2}-1\right) \delta r_{o},
\end{gathered}
$$

to first order in the perturbation. Taking into account (12) and (13), and solving for $\phi_{i}^{1}$ and $\phi_{o}^{1}$, we obtain

$$
\begin{aligned}
& \phi_{i}^{1}\left(r_{i}\right)=\left\{M \frac{\sigma}{r_{i}^{2}}\left(n^{2}-1\right)+M \rho \Omega^{2} r_{i}-\left.\partial_{r} \phi^{0}\right|_{r_{i}}\right\} \zeta_{i}, \\
& \phi_{o}^{1}\left(r_{o}\right)=\left\{-M \frac{\sigma}{r_{o}^{2}}\left(m^{2}-1\right)+M \rho \Omega^{2} r_{o}-\left.\partial_{r} \phi^{0}\right|_{r_{o}}\right\} \zeta_{o},
\end{aligned}
$$

which implies

$$
\begin{aligned}
& D_{n}=\frac{1}{\left[1-\left(r_{o} / r_{i}\right)^{2 n}\right] r_{i}^{n}} P_{i} \zeta_{i}, \\
& D_{m}=\frac{1}{\left[1-\left(r_{i} / r_{o}\right)^{2 m}\right] r_{o}^{m}} P_{o} \zeta_{o},
\end{aligned}
$$

with

$$
\begin{aligned}
& P_{i}=M\left\{\frac{\sigma}{r_{i}^{2}}\left(n^{2}-1\right)+\rho \Omega^{2} r_{i}-\frac{A}{M r_{i}}\right\}, \\
& P_{o}=M\left\{-\frac{\sigma}{r_{o}^{2}}\left(m^{2}-1\right)+\rho \Omega^{2} r_{o}-\frac{A}{M r_{o}}\right\} .
\end{aligned}
$$

The growth rate of the perturbation is derived from Darcy's law, which relates the time derivative of the radial coordinate to the spatial derivative of $\phi$ in the radial direction. We obtain

$$
\begin{aligned}
& \delta \dot{r}_{i}=\left.\partial_{r r} \phi^{0}\right|_{r_{i}} \delta r_{i}+\left.\partial_{r}\left(\phi_{i}^{1} e^{i n \theta}+\phi_{o}^{1} e^{i m \theta}\right)\right|_{r_{i}}, \\
& \delta \dot{r}_{o}=\left.\partial_{r r} \phi^{0}\right|_{r_{o}} \delta r_{o}+\left.\partial_{r}\left(\phi_{i}^{1} e^{i n \theta}+\phi_{o}^{1} e^{i m \theta}\right)\right|_{r_{o}} .
\end{aligned}
$$

The overdot stands for a time derivative. Substituting the velocity potentials by their expressions (10) and (15) and taking spatial derivatives, these two equations can be rewritten in the form

$$
\begin{aligned}
\dot{\zeta}_{i}= & -\frac{A}{r_{i}^{2}} \zeta_{i}-\frac{n}{r_{i}} \frac{1+\left(r_{i} / r_{o}\right)^{2 n}}{1-\left(r_{i} / r_{o}\right)^{2 n}} P_{i} \zeta_{i} \\
& -\frac{2 m}{r_{i}} \frac{e^{i(m-n) \theta}}{\left(r_{i} / r_{o}\right)^{m}-\left(r_{o} / r_{i}\right)^{m}} P_{o} \zeta_{o}, \\
\dot{\zeta}_{o}= & -\frac{A}{r_{o}^{2}} \zeta_{o}-\frac{2 n}{r_{o}} \frac{e^{i(n-m) \theta}}{\left(r_{o} / r_{i}\right)^{n}-\left(r_{i} / r_{o}\right)^{n}} P_{i} \zeta_{i} \\
& -\frac{m}{r_{o}} \frac{1+\left(r_{o} / r_{i}\right)^{2 m}}{1-\left(r_{o} / r_{i}\right)^{2 m}} P_{o} \zeta_{o} .
\end{aligned}
$$

Since the amplitudes $\zeta_{i}$ and $\zeta_{o}$ cannot depend on the azimuthal angle, two possibilities arise:

(i) The term with the exponential is zero in the expressions above [Eqs. (28) and (29)]. This occurs in the limit of a large separation between the two interfaces, $r_{o} \gg r_{i}$, which will be discussed in detail later.

(ii) $m=n$, independently of the ratio between $r_{o}$ and $r_{i}$. This case is discussed next.

The condition $m=n$ leads to a dispersion relation of the form 


$$
\left(\begin{array}{l}
\dot{\zeta}_{i} \\
\dot{\zeta}_{o}
\end{array}\right)=\left(\begin{array}{ll}
a_{11} & a_{12} \\
a_{21} & a_{22}
\end{array}\right)\left(\begin{array}{l}
\zeta_{i} \\
\zeta_{0}
\end{array}\right)
$$

where

$$
\begin{aligned}
& a_{11}=-\frac{A}{r_{i}^{2}}-\frac{n}{r_{i}} \frac{1+\left(r_{i} / r_{o}\right)^{2 n}}{1-\left(r_{i} / r_{o}\right)^{2 n}} P_{i}, \\
& a_{12}=-\frac{2 n}{r_{i}} \frac{1}{\left(r_{i} / r_{o}\right)^{n}-\left(r_{o} / r_{i}\right)^{n}} P_{o}, \\
& a_{21}=-\frac{2 n}{r_{o}} \frac{1}{\left(r_{o} / r_{i}\right)^{n}-\left(r_{i} / r_{o}\right)^{n}} P_{i}, \\
& a_{22}=-\frac{A}{r_{o}^{2}}-\frac{n}{r_{o}} \frac{1+\left(r_{o} / r_{i}\right)^{2 n}}{1-\left(r_{o} / r_{i}\right)^{2 n}} P_{o} .
\end{aligned}
$$

This relation shows that, in the generic case $r_{i} \sim r_{o}$, the two interfaces are coupled to each other through the pressure field, already at a linear level.

To determine whether the perturbation at the interfaces grows or decays in time we have to diagonalize the dispersion relation (30). The corresponding eigenvalues $\omega_{ \pm}(n)$ determine the growth rates of the normal modes. We find:

$$
\begin{aligned}
\omega_{ \pm}= & \frac{1}{2}\left(a_{11}+a_{22}\right) \pm \frac{1}{2}\left[\left(a_{11}+a_{22}\right)^{2}\right. \\
& \left.+4\left(a_{12} a_{21}-a_{11} a_{22}\right)\right]^{1 / 2} .
\end{aligned}
$$

These growth rates correspond to the following normal modes of perturbation:

$$
\begin{aligned}
& \delta \mathbf{r}_{+}=\zeta_{i} e^{i n \theta}\left(\begin{array}{c}
1 \\
\frac{\omega_{+}-a_{11}}{a_{12}}
\end{array}\right), \quad \text { for which } \frac{\delta \dot{r}_{+}}{\delta r_{+}}=\omega_{+}, \\
& \delta \mathbf{r}_{-}=\zeta_{0} e^{i n \theta}\left(\begin{array}{c}
\frac{\omega_{-}-a_{22}}{a_{21}} \\
1
\end{array}\right), \quad \text { for which } \frac{\delta \dot{r}_{-}}{\delta r_{-}}=\omega_{-} .
\end{aligned}
$$

The behavior of these two modes depends on the sign of the component $\left(\omega_{ \pm}-a_{i i}\right) / a_{i j}$. If this component is real and positive, the perturbations at the two interfaces are in phase, and the mode is a bending mode. If this component is real and negative, the perturbations have a phase difference of $\pi / 2$ rads and the mode is a squeezing mode. This component can also have a nonzero imaginary part, which would lead to a spatio-temporal oscillation of the perturbations at the two interfaces.

\section{Linear stability of a very wide annulus $\left(r_{o} \gg r_{i}\right)$}

It is interesting to analyze the stability of the interfaces in the limit of a large separation distance, $r_{o} \gg r_{i}$. We have

$$
\begin{aligned}
& a_{11} \simeq-\frac{A}{r_{i}^{2}}-\frac{n}{r_{i}} P_{i}, \\
& a_{22} \simeq-\frac{A}{r_{o}^{2}}+\frac{m}{r_{o}} P_{o}, \\
& a_{12} \simeq 0,
\end{aligned}
$$

$$
a_{21} \simeq 0 .
$$

We see that the nondiagonal terms in Eq. (30) vanish and the stability of the leading and trailing interface becomes independent. As the nondiagonal terms are negligible, the condition $m=n$ does not necessarily hold, and we are led to distinguish again between the azimuthal wavenumbers $n$ and $m$. The growth rates, Eq. (35), are given now by

$\omega_{+} \simeq a_{11} \simeq M\left\{-\frac{\sigma}{r_{i}^{3}} n\left(n^{2}-1\right)-\rho \Omega^{2} n+\frac{A}{M r_{i}^{2}}(n-1)\right\}$,

$\omega_{-} \simeq a_{22} \simeq M\left\{-\frac{\sigma}{r_{o}^{3}} m\left(m^{2}-1\right)+\rho \Omega^{2} m-\frac{A}{M r_{o}^{2}}(m+1)\right\}$,

and the corresponding modes by

$$
\begin{aligned}
& \delta \mathbf{r}_{+} \simeq \zeta_{i} e^{i n \theta}\left(\begin{array}{l}
1 \\
0
\end{array}\right), \\
& \delta \mathbf{r}_{-} \simeq \zeta_{o} e^{i n \theta}\left(\begin{array}{l}
0 \\
1
\end{array}\right) .
\end{aligned}
$$

In this limit, the first mode is an exclusive perturbation on the inner interface, and the second mode an exclusive perturbation on the outer interface. The growth rate of the perturbation of the inner interface, Eq. (42), reproduces the classic result of Paterson ${ }^{7}$ for air displacing oil in a circular geometry, in which the instability is driven by the viscosity contrast between the two fluids, with surface tension stabilizing modes of short wavelength (large $n$ ). Here there is an additional term accounting for centrifugal forcing, which stabilizes due to the fact that at this interface the outer fluid (oil) is the densest. The growth rate of the perturbation of the outer interface, Eq. (43), on the other hand, reproduces the results of Schwartz ${ }^{8}$ and Carrillo et al. ${ }^{9}$ for oil displacing air in the presence of centrifugal forcing. In this case the instability is driven by the density contrast between the two fluids, while the viscosity contrast and the interfacial tension have a stabilizing effect.

\section{E. Linear stability of a very thin annulus $\left(r_{o} \simeq r_{i}\right)$}

We study the linear stability of the circular interfaces as the radially spreading annulus becomes very thin and the two interfaces get very close to each other. This is the opposite limit of the previous case, and the coupling of the two interfaces is expected to play an important role here. This limit can only be carried up to the point at which the thickness $r_{o}-r_{i}$ of the annulus becomes comparable with the gap thickness $b$ of the cell, since at this point three-dimensional effects become determinant and the Hele-Shaw approximation fails.

The limit of a very thin annulus must be taken with some care because $r_{i} \rightarrow r_{o}$, while $L^{2}=r_{o}^{2}-r_{i}^{2}$ remains a constant of the motion. To take both aspects into consideration at the same time, we define a small parameter $\epsilon=L / r_{o}$, such that 


$$
\left(\frac{r_{i}}{r_{o}}\right)^{2}=1-\left(\frac{L}{r_{o}}\right)^{2}=1-\epsilon^{2} \rightarrow 1, \quad \text { as } \quad \epsilon \rightarrow 0,
$$

corresponding to $r_{o} \rightarrow \infty$ with $L$ constant. We now consider the constant $A$ in the approximation of perfect wetting

$$
A=M \frac{(1 / 2) \rho \Omega^{2} L^{2}-\sigma\left(1 / r_{i}+1 / r_{o}\right)}{\ln \left(r_{o} / r_{i}\right)},
$$

and expand $A, P_{i}, P_{o}$ in powers of $\epsilon$. After a lengthy calculation, one obtains

$$
\begin{aligned}
& a_{11}=M\left\{-\frac{\sigma}{L^{3}}\left[8 \epsilon^{-1}+\mathcal{O}(\epsilon)\right]-\rho \Omega^{2}\left[\frac{1}{6} \epsilon^{2}+\mathcal{O}\left(\epsilon^{4}\right)\right]\right\}, \\
& a_{22}=M\left\{\frac{\sigma}{L^{3}}\left[8 \epsilon^{-1}+\mathcal{O}(\epsilon)\right]+\rho \Omega^{2}\left[\frac{1}{6} \epsilon^{2}+\mathcal{O}\left(\epsilon^{4}\right)\right]\right\}, \\
& a_{12}=M\left\{\frac{\sigma}{L^{3}}\left[8 \epsilon^{-1}+\mathcal{O}(\epsilon)\right]+\rho \Omega^{2}\left[1+\frac{1}{6} \epsilon^{2}+\mathcal{O}\left(\epsilon^{4}\right)\right]\right\},
\end{aligned}
$$

$a_{21}=M\left\{-\frac{\sigma}{L^{3}}\left[8 \epsilon^{-1}+\mathcal{O}(\epsilon)\right]+\rho \Omega^{2}\left[1-\frac{1}{6} \epsilon^{2}+\mathcal{O}\left(\epsilon^{4}\right)\right]\right\}$.

The linear growth rates of the normal modes, given by Eq. (35), now become:

$$
\omega_{ \pm} \simeq \pm M \rho \Omega^{2},
$$

to leading order in $\epsilon$. The leading contribution is in the nondiagonal terms, $a_{12}$ and $a_{21}$, which account for the coupling of the two interfaces. Notice that the growth rates are independent of $n$. The corresponding modes are

$$
\begin{aligned}
& \delta \mathbf{r}_{+} \simeq \zeta_{i} e^{i n \theta}\left(\begin{array}{l}
1 \\
1
\end{array}\right), \\
& \delta \mathbf{r}_{-} \simeq \zeta_{o} e^{i n \theta}\left(1+\frac{1}{4} \frac{\rho \Omega^{2} L^{3}}{\sigma} \epsilon\right) . \\
& 1
\end{aligned}
$$

The mode $\delta \mathbf{r}_{+}$is a pure bending mode. The perturbations at the two interfaces are in phase and have identical amplitude as a consequence of the strong coupling of the interfaces. The mode $\delta \mathbf{r}_{-}$is also a bending mode because the perturbations at the two interfaces are in phase, but now the amplitude of the perturbation at the trailing interface is larger than the amplitude of the perturbation at the leading interface. This can give rise to the formation of trapped fluid regions right before the two interfaces meet each other, similarly to what would be observed for a squeezing mode. We will call $\delta \mathbf{r}_{-}$an enhanced bending mode.

The linear growth rate of the pure bending mode is positive for all $n$. Hence, the annulus is always unstable against bending, when it becomes sufficiently thin. The picture on top of Fig. 4 provides an example of slight destabilization by a pure bending mode in prewet conditions. The linear growth rate of the enhanced bending mode shows that this mode always decays, so that upon thinning the annulus becomes linearly stable against this kind of perturbation.
The different behavior of the normal modes, however, must be rationalized in terms of the evolution of the base state itself. Notice that the growth-decay rate of the two modes is given by the reciprocal of $\tau=12 \mu /\left(\rho \Omega^{2} b^{2}\right)$, which is precisely the time scale of a rotating Hele-Shaw system. ${ }^{9}$ This has important consequences on the behavior of the normal modes. To see why, consider conditions of perfect wetting - which were assumed in the derivation of the preceding results. The velocity of the leading interface is $v_{o}$ $=A / r_{o} \simeq M \rho \Omega^{2} r_{o}=r_{o} / \tau$. Therefore $r_{o}$ grows exponentially with time in prewet conditions as $\exp (t / \tau)$. Given that $L^{2}$ $=r_{o}^{2}-r_{i}^{2}$ is a constant of the motion, the radius $r_{i}$ follows this same behavior. These predictions have been confirmed experimentally in Ref. 11. Finally, it is not difficult to show that the separation of the interfaces $r_{o}-r_{i}$ goes to zero as $\exp (-t / \tau)$, i.e., with the same constant $\tau$. Now, the amplitude of the mode $\delta \mathbf{r}_{+}$must be compared with the radii of the circular interfaces. Only if the amplitude grows at higher rate than the two radii will the morphological instability develop at finite time. It turns out that they grow at exactly the same rate $\tau$, which is equivalent to saying that their relative amplitude remains constant in time, and $\delta \mathbf{r}_{+}$is marginal with respect to the base state. The overall behavior can be regarded as a spatial rescaling at successive times of the perturbed thin annulus. The amplitude of the mode $\delta \mathbf{r}_{-}$, on the other hand, must be compared with the separation of the interfaces. If the unperturbed interfaces approach each other at higher rate than the mode decays, there is pinching of the interfaces at finite time-even though the amplitude of the mode decays in time. It turns out that they all decay at exactly the same rate $\tau$, so that $\delta \mathbf{r}_{-}$is marginal with respect to the base state. In conclusion, none of the two normal modes is expected to produce a morphological instability of the thin annulus in prewet conditions at finite time.

In dry conditions the situation is different, since the radii of the circular interfaces do not grow exponentially with time, but linearly, and their velocity is significantly lower than in prewet conditions. ${ }^{11}$ The growth-decay rate of the normal modes could be comparatively higher. These questions are addressed in the next section.

\section{F. Numerical integration of the equations and comparison with experimental results}

To compare the predictions of the linear stability analysis with our experimental observations, we have carried out a numerical integration of the coupled equations [Eqs. (28) and (29)].

The first point to consider is that the base state in the linear stability analysis (circular interfaces of radii $r_{o}$ and $r_{i}$ ) is actually evolving in time. This makes the linear growth rates of the normal modes dependent on time, and the relaxation or growth of the modes not truly exponential. This is usual in circular geometry. For example, in the classic experiments of Paterson ${ }^{7}$ with air displacing oil in a motionless circular Hele-Shaw cell, the linear evolution of the unstable interface proceeds through a cascade of modes, with increasing participation of higher $n$ modes as time progresses.

In our case the linear evolution of the interfaces is more 


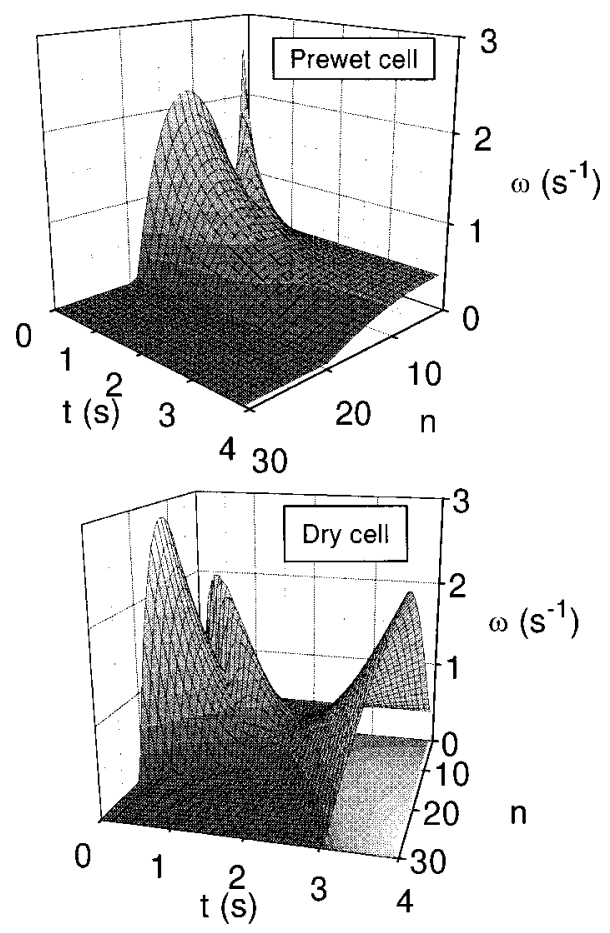

FIG. 10. Linear growth rate of the different modes $n$ as a function of time for prewet and dry conditions. The parameters used in the numerical integration are $b=0.81 \mathrm{~mm}, L_{0}=30 \mathrm{~mm}, \Omega=180 \mathrm{rev} / \mathrm{min}, \rho=1000 \mathrm{~kg} / \mathrm{m}^{3}$, $\nu=50 \mathrm{~mm}^{2} / \mathrm{s}, \sigma=20.7 \mathrm{mN} / \mathrm{m}$.

complicated, because the linear growth rates $\omega_{ \pm}$depend on the radii $r_{o}$ and $r_{i}$ through the matrix elements $a_{i j}$. To calculate the time dependence of the linear growth rates, the annulus is originally defined as having inner radius $R_{A}$ (the radius of the central orifice of the top plate) and outer radius $L_{0}$. The subsequent radii of the stable circular interfaces, $r_{i}$ and $r_{o}$, and the matrix elements $a_{i j}$ are computed as a function of time using the equations for the stable displacement derived in our previous work. ${ }^{11}$

(i) In prewet conditions we use Eqs. (10) and (11), with $\theta_{D i} \simeq \theta_{D o} \simeq 0$ and $L^{2}=r_{o}^{2}-r_{i}^{2}$ practically constantbecause the relative thicknesses verify $\alpha_{i} \simeq \alpha_{o}$. Since $L$ remains constant, $\alpha_{i}$ and $\alpha_{o}$ have no influence on $r_{i}, r_{o}$.

(ii) In dry conditions we follow the same procedure only in the first time steps, until the velocity $v_{i}$ of the trailing interface becomes larger than the velocity given by the empirical scaling relation $v=0.1(\sigma / \mu)$ $\times\left(b / L_{0}\right)^{2} S^{5 / 4}$. From this time step on, we determine $v_{i}$ from the scaling relation. Next, $v_{i}$ is used to determine $r_{i}$ and the function $A$ given by $A=-v_{i} r_{i} / M$. Finally, the radius $r_{o}$ of the leading interface is determined from $r_{o}^{2}=L^{2}+r_{i}^{2}$, taking into account that in dry conditions $\alpha_{o}=0$ and $\alpha_{i} \neq 0$, which makes $L$ a decreasing function of $r_{i}$. The thickness $\alpha_{i}$ is computed from $v_{i}$ through Reinelt's formulas for a circular interface. ${ }^{12}$

The time dependence of the linear growth rates is shown in Fig. 10. Since it is difficult to keep track of the two modes separately, we plot only the mode of highest growth rate at each time. In both prewet and dry conditions, the figure shows two maxima at $t=0$ which decay in time at short times. Of these two maxima, the one for low $n$ is associated with the trailing interface. This interface is always unstable at the beginning because of its large initial velocity $(v$ $\sim 1 / r)$, but can only accommodate perturbations of very low wave number due to its small radius. The other initial maximum, for intermediate $n$, is associated with the leading interface. The stability of this interface is not significantly affected by the trailing interface at short times, and thus it is governed by Eq. (43), i.e., by the interplay between the destabilizing effect of density contrast and the stabilizing effect of both viscosity contrast and surface tension.

The influence of wetting conditions appears at later times, when the two original maxima in Fig. 10 have nearly disappeared. In prewet conditions the radii of the interfaces grow exponentially in time. As a result, the linear growth rates remain small and nearly constant in time. In dry conditions, on the other hand, the radii of the two interfaces grow linearly in time, and the growth rates of the unstable modes rise sharply in time.

The fact that the linear growth rates depend on time means that the relaxation or growth of the normal modes for a wavenumber $n$ is not proportional to $\exp \left[\omega_{ \pm}(n)\right] t$. Rather, a direct integration of the linearized equations for the evolution of the normal modes, (28) and (29), leads to

$$
\delta r_{ \pm}(t)=\delta r_{ \pm}(0) \exp \left[\int_{0}^{t} \omega_{ \pm}(n) d t^{\prime}\right]
$$

provided that $\int_{0}^{t} \omega_{ \pm}(n) d t^{\prime}>0$, which means that the disturbance grows and the modes are unstable. The number of fingers at the end of the linear regime is typically given by the mode $n$ which has grown to the largest amplitude, not by the instantaneous fastest growing mode.

With these considerations in mind, we have computed the time dependence of the amplitudes of the different modes from a numerical integration of Eqs. (28) and (29). Following Cardoso and Woods ${ }^{10}$ and Miranda and Widom, ${ }^{13}$ we assume the presence of a constant level of noise in the experiment, which perturbs each mode $n$ with the same amplitude $|\delta r(0)|$. The calculation requires a specific value of the temporal extent of the linear regime, which is not readily experimentally accessible. We have decided to carry out the numerical integration up to a time $t_{f}=0.3 \tau$, where $\tau$ is the natural time scale for radial Hele-Shaw displacements under centrifugal forcing and has been defined in Sec. III E. This choice, although arbitrary, is based on empirical results obtained for experiments of fingering of a circular drop under centrifugal forcing in the same experimental setup. ${ }^{9}$ In these experiments the instability developed systematically only after a latency time $t / \tau=0.3$. We take this scaling quantity as indicative of the duration of the linear regime.

The result of the calculation is shown in Fig. 11. The relative amplitude of the different modes is plotted as a function of time, for prewet and dry conditions. $A_{0}$ is the amplitude at $t=0$ (amplitude of the noise in the initial condition). Again, only the mode that has the largest amplitude is shown at each time. It is clear from this result that the time scale for 

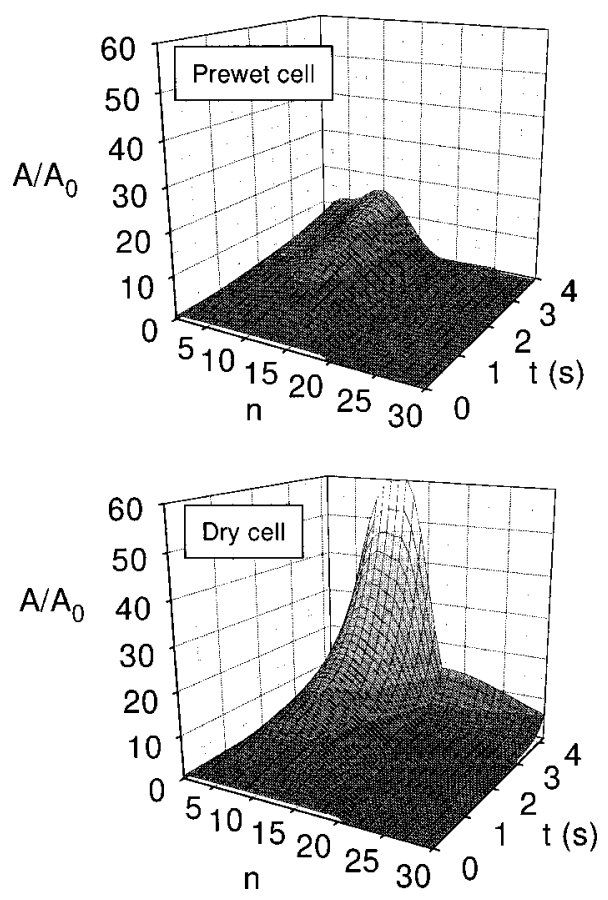

FIG. 11. Relative amplitude of the different modes $n$ as a function of time for prewet and dry conditions. The parameters used in the numerical integration are the same as in Fig. 10.

development of the instability is very different in prewet and dry conditions. Under equivalent experimental conditions, the instability in a prewet cell will possibly not develop appreciably during the experiment, while in a dry cell it will clearly do so in almost all instances. In both prewet and dry conditions the wave number of maximum amplitude increases slightly with time, predicting a cascade towards modes of larger $n$ as time increases. These predictions are in good agreement with our experimental observations.

The preceding analysis has been carried out systematically in the range of parameters explored experimentally in dry conditions. We have determined numerically the wave number $n$ of the mode which grows to its largest amplitude at the first unstable interface, as a function of rotation rate $\Omega$ and initial radius $L_{0}$. The result is presented in Fig. 12 (top). The region from the lower-left corner to the middle of the diagram corresponds to the leading interface, and the region from the middle to the upper-right corner to the trailing interface. Clearly, in view of the assumptions required to compute it, this diagram can only be considered from a qualitative point of view. In particular, the boundary between the two regions is strongly dependent on the arbitrary choice of the total time of integration $t_{f}$.

Nevertheless, the trend exhibited by the lines of constant $n$ reproduces well our experimental observations, which are also summarized on Fig. 12 (bottom). Experimentally, the values of $n$ have been determined in the following way. In the case of a leading interface, which is the case encountered most often, $n$ is the number of fingers observed in the developed pattern. As discussed before, this number is a good estimate of the dominant wave number emerging from the linear regime, due to the fact that at the leading interface the nonlinear mechanisms capable of modifying the number of
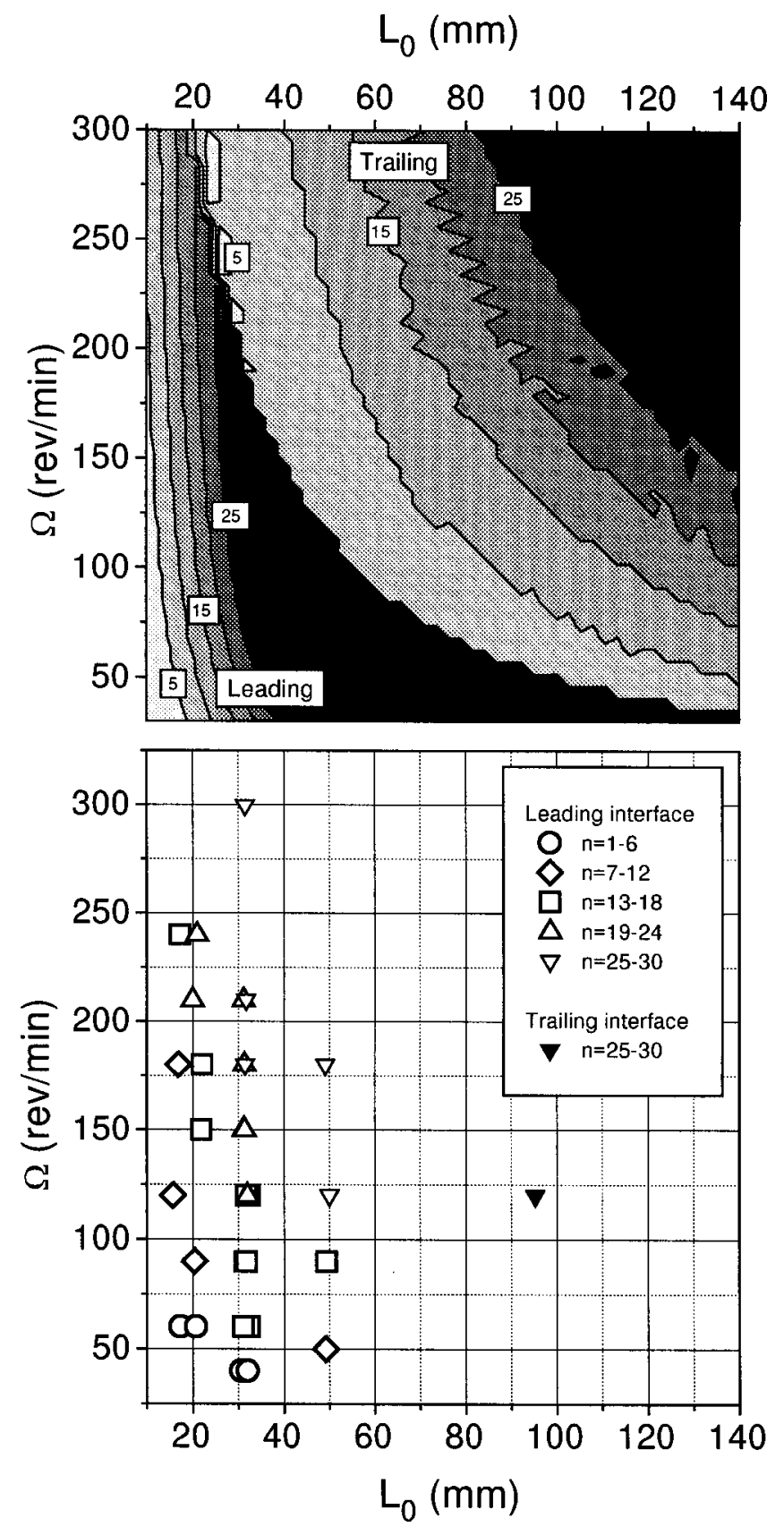

FIG. 12. Lines of constant wave number $n$, in the parameter space $\Omega$ (rotational frequency) vs $L_{0}$ (initial radius of the oil drop), for displacements in dry conditions, with $b=0.81 \mathrm{~mm}, \nu=50 \mathrm{~mm}^{2} / \mathrm{s}$, and $\sigma=20.7 \mathrm{mN} / \mathrm{m}$. Top: prediction of the linear stability analysis for the mode that grows to largest amplitude. Bottom: Experimental results. Both diagrams comprise two different regions; one from the bottom-left corner to the middle of the diagram, in which the first unstable interface is the leading interface, and another, from the middle to the top-right corner, in which the first unstable interface is the trailing interface.

fingers (finger competition or tip splitting) do not seem to be operative. In the less frequent case of analyzing a trailing interface, the number $n$ is taken as the wave number of the mode that displays the peak of largest amplitude in the power spectrum, at the end of the linear regime. The extent of the linear regime is determined by the condition that the amplitude of the ripples in the radial direction is smaller than their azimuthal wavelength. 


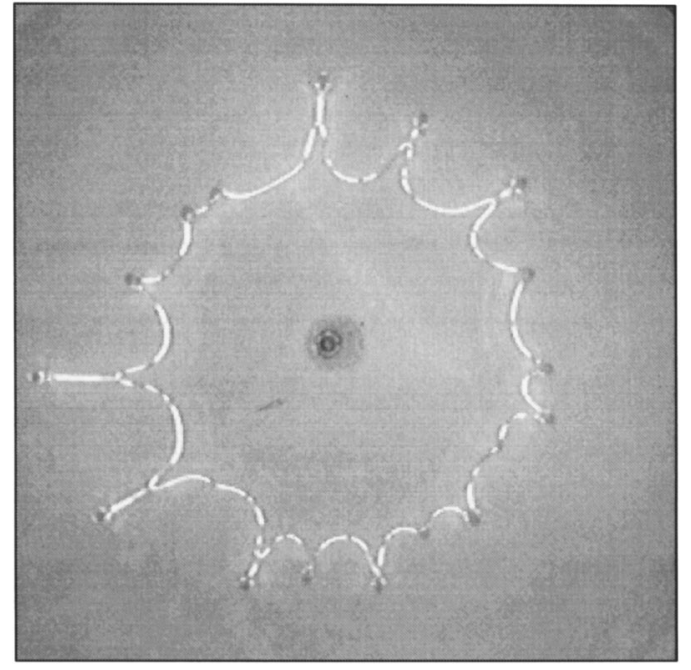

FIG. 13. Late stages in the evolution of a rotating annulus in a dry cell. The picture shows a cord of liquid which emits droplets in the radial direction through the formation of thin fluid filaments at the tip of outgrowing fingers. In this experiment the fluid is silicone oil $50, \Omega=120 \mathrm{rev} / \mathrm{min}, b$ $=1.94 \mathrm{~mm}, L_{0}=30 \mathrm{~mm}$, and the picture is taken at $t=2.6 \mathrm{~s}$.

The dependence of $n$ on initial radius $L_{0}$, which has been discussed in connection with Fig. 6, is observed here to be general; for small $L_{0}$ the first unstable interface is the leading interface, and $n$ is small. As $L_{0}$ increases, $n$ increases correspondingly. Then $L_{0}$ reaches a threshold value at which the leading interface is replaced by the trailing interface, and the number $n$ becomes small again. Our observations show that the threshold value of $L_{0}$, which separates the onset of instability at one or another interface, becomes lower as $\Omega$ increases.

\section{G. Other experimental observations}

An interesting feature of the fingering patterns observed in the present experiments is the radial emission of droplets in the deep nonlinear regime. This observation might be related to the problems of pearling and pinching in Hele-Shaw flows, which have been studied theoretically and numerically in recent years. ${ }^{14-17}$ In our experiment, the emission of droplets occurs in very thin annuli, and in dry conditions it is accompanied by the formation of thin fluid filaments at the tip of radially outgrowing fingers, as shown in Fig. 13. It is interesting to point out that the radial growth velocity of a fluid filament is appreciably larger than the radial velocity of the finger from which it formed, as shown in the sequence of pictures in Fig. 14.

Given that the thicknesses in the radial direction and in the direction perpendicular to the glass plates are comparable in this case, it is not clear that the secondary instability giving rise to the formation of fluid filaments and the emission of droplets can be analyzed within the framework of the Hele-Shaw approximation.

Ignoring the real possibility of non-Hele-Shaw effects, our experimental observations could be analyzed in the framework of the nonlinear theory developed by Goldstein, Pesci, and Shelley, ${ }^{14}$ in which they use the lubrication theory for Hele-Shaw flows to study the pinching of the neck of a
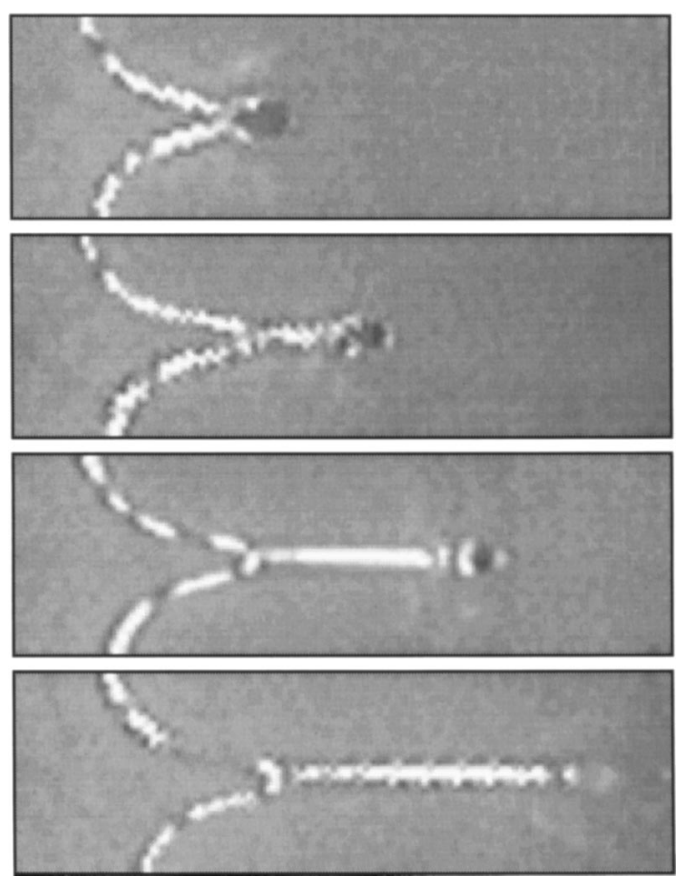

FIG. 14. Sequence of pictures taken from the experiment shown in Fig. 13, demonstrating the formation of a droplet at the tip of an outgrowing finger, and its fast departure from the finger through the formation of a thin fluid filament. The first picture is taken at $t=2.2 \mathrm{~s}$, and the interval between pictures is $0.2 \mathrm{~s}$.

pendant drop as well as a driven jet. Our centrifugally driven necks and droplets seem somewhere between these two cases. This analysis, which goes beyond the scope of the present paper, is currently in progress.

\section{SUMMARY}

We have studied the stability of an annular layer of fluid confined in a circular Hele-Shaw cell and subjected to centrifugal forcing. The annular configuration is interesting because the two interfaces can be made simultaneously unstable. The instability of the leading interface is driven by the density contrast in the presence of centrifugal forcing. The instability of the trailing interface is driven by the large viscosity contrast.

Our experimental results have shown that flows in a cell prewet with an oil coating are highly stable in the range of parameters explored. On the other hand, in a dry cell the flows are unstable. In this case the instability develops first at the leading interface, or simultaneously at both interfaces if the annulus becomes very thin. This kind of instability generates fingers, which grow radially. The number of fingers is the same at both interfaces when the annulus is very thin. Deep in the nonlinear regime, these fingers are not seen to tip split or compete. Only for initially thick annuli does the instability develop first at the trailing interface, and the fingers at this interface exhibit tip splitting.

We have carried out a linear stability analysis of the problem and shown that the stability of the two interfaces is coupled through the pressure field, already at a linear level.

We have studied the limit of a thick annulus, for which the two interfaces decouple. The result of the linear stability 
analysis reduces in this limit to the classical dispersion relations of the circular interfaces in the presence of centrifugal forcing; at the leading interface (oil displacing air) the instability is driven by the density contrast between the fluids, in competition with the stabilizing effects of viscosity contrast and interfacial tension. At the trailing interface (air displacing oil) the instability is driven by the viscosity contrast between the two fluids, and density contrast and interfacial tension stabilize.

We have also studied the limit of a thin annulus, which is gradually reached as the annulus spreads radially in the circular cell. In this limit and in prewet conditions the coupling of the two interfaces is shown to lead to two possible modes of perturbation; a bending mode $\delta \mathbf{r}_{+}$, unstable, which in the linear regime grows exponentially at a rate $1 / \tau$ for all wave numbers $n$, and an enhanced bending mode $\delta \mathbf{r}_{-}$, stable, which decays at a rate $-1 / \tau$. We have shown that the two modes are marginally stable with respect to the radial displacement of the annulus (in the case of $\delta \mathbf{r}_{+}$) and to the radial thinning of the annulus (in the case of $\delta \mathbf{r}_{-}$). Consequently they are not expected to produce a morphological instability of the thin annulus at finite time. This is not the case in dry conditions, for which the radii of the circular interfaces grow comparatively slower.

Between these two limits, the linear stability of the annulus has been addressed by a numerical integration of the coupled equations for the linear growth rates of the normal modes. The result shows a mode-to-mode cascade to larger $n$ as time progresses. The effect of wetting conditions has also been taken into account in the numerical integration, through the unperturbed velocity fields derived in Ref. 11 . We have found that the time scale for amplification of infinitesimal perturbations is much shorter in a dry cell than in a cell previously coated with oil, in agreement with our experimental observations.

Although the predictions of the linear stability analysis cannot be easily verified experimentally at a quantitative level, we have succeeded in showing that the general trends of the number of fingers predicted at the end of the linear regime, in the parameter space of rotational frequency vs initial radius, are in good qualitative agreement with our experimental observations.
Finally, we have observed a secondary bifurcation of the fingers in very thin annuli, with the formation of fluid droplets and their emission at large velocity in the radial direction.

\section{ACKNOWLEDGMENTS}

We thank Jaume Casademunt for fruitful discussions. This work has received the financial support of the Dirección General de Enseñanza Superior (DGES, Spain), Projects PB96-0378-C02-01 and PB97-0906, and has been developed within the framework of the Training and Mobility of Researchers program of the European Community (network project ERBFMRX-CT96-0085).

${ }^{1}$ P. G. Saffman and G. I. Taylor, "The penetration of a fluid into a porous medium or Hele-Shaw cell containing a more viscous liquid,' Proc. R. Soc. London, Ser. A 245, 312 (1958).

${ }^{2}$ G. M Homsy, "Viscous fingering in porous media,' Annu. Rev. Fluid Mech. 19, 271 (1987).

${ }^{3}$ K. J. Ruschak, 'Coating flows,'” Annu. Rev. Fluid Mech. 17, 65 (1985).

${ }^{4}$ D. Bensimon, L. P. Kadanoff, S. Liang, B. I. Shraiman, and C. Tang, "Viscous flows in two dimensions,"' Rev. Mod. Phys. 58, 977 (1986).

${ }^{5}$ P. Pelcé, Dynamics of curved fronts (Academic, San Diego, CA, 1988).

${ }^{6}$ K. V. McCloud and J. V. Maher, "Experimental perturbations to Saffman-Taylor flow,' Phys. Rep. 260, 139 (1995).

${ }^{7}$ L. Paterson, "Radial fingering in a Hele-Shaw cell,', J. Fluid Mech. 113, 513 (1981)

${ }^{8}$ L. W. Schwartz, "Instability and fingering in a rotating Hele-Shaw cell or porous medium,', Phys. Fluids A 1, 167 (1989).

${ }^{9}$ L1. Carrillo, F. X. Magdaleno, J. Casademunt, and J. Ortín, “Experiments in a rotating Hele-Shaw cell,'” Phys. Rev. E 54, 6260 (1996).

${ }^{10}$ S. S. S. Cardoso and A. W. Woods, "The formation of drops through viscous instability,', J. Fluid Mech. 289, 351 (1995).

${ }^{11} \mathrm{Ll}$. Carrillo, J. Soriano, and J. Ortín, "Radial displacement of a fluid annulus in a rotating Hele-Shaw cell,' Phys. Fluids 11, 778 (1999).

${ }^{12}$ D. A. Reinelt, "Interface conditions for two-phase displacement in HeleShaw cells,' J. Fluid Mech. 183, 219 (1987).

${ }^{13}$ J. A. Miranda and M. Widom, "Radial fingering in a Hele-Shaw cell: a weakly nonlinear analysis,' Physica D 120, 315 (1998).

${ }^{14}$ R. E. Goldstein, A. I. Pesci, and M. J. Shelley, "Topology transitions and singularities in viscous flows,' Phys. Rev. Lett. 70, 3043 (1993).

${ }^{15}$ P. Constantin, T. F. Dupont, R. E. Goldstein, L. P. Kadanoff, M. Shelley, and S.-M. Zhou, "Droplet breakup in a model of the Hele-Shaw cell,", Phys. Rev. E 47, 4169 (1993)

${ }^{16}$ T. F. Dupont, R. E. Goldstein, L. P. Kadanoff, and S.-M. Zhou, "Finitetime singularity formation in Hele-Shaw systems,' Phys. Rev. E 47, 4182 (1993).

${ }^{17}$ D. Papageorgiou, "On the break-up of viscous liquid threads," Phys. Fluids 7, 1529 (1995) 